\title{
Les épreuves du corps en littérature. Les cas de la
} Chine et du Japon

On Body Trials in Literature. China and Japan As Case-Studies

Cécile Sakai, Gérard Siary et Victor Vuilleumier

\section{(2) OpenEdition}

\section{Journals}

Édition électronique

URL : http://journals.openedition.org/extremeorient/386

DOI : 10.4000/extremeorient.386

ISBN : 978-2-84-292-449-2

ISSN : 2108-7105

Éditeur

Presses universitaires de Vincennes

\section{Édition imprimée}

Date de publication : 1 octobre 2015

Pagination : $5-20$

ISBN : 978--84292-447-8

ISSN : 0754-5010

\section{Référence électronique}

Cécile Sakai, Gérard Siary et Victor Vuilleumier, « Les épreuves du corps en littérature. Les cas de la Chine et du Japon », Extrême-Orient Extrême-Occident [En ligne], 39 | 2015, mis en ligne le 01 août 2016, consulté le 24 septembre 2020. URL : http://journals.openedition.org/extremeorient/386 ; DOI : https://doi.org/10.4000/extremeorient.386 


\title{
Les épreuves du corps en littérature Les cas de la Chine et du Japon
}

\author{
Cécile Sakai, Gérard Siary et Victor Vuilleumier
}

\begin{abstract}
«Le corps humain référent des descriptions devient métonymie ou métaphore de l'ordre du monde. Le corps, métaphore de l'ordre rêvé, est dans ses défaillances métaphore de tous les désordres ${ }^{1}$. »
\end{abstract}

Le présent numéro d'Extrême-Orient Extrême-Occident, "Corps souffrants dans les littératures de la Chine et du Japon au $\mathrm{XX}^{\mathrm{e}}$ siècle », s'inscrit dans le prolongement du programme transversal 2014-2018 du Centre de recherches sur les civilisations de l'Asie orientale (en partenariat privilégié avec l'Université de Genève) : «Imaginaires du corps et des identités dans les littératures de l'Asie orientale au $\mathrm{XX}^{\mathrm{e}}$ siècle $^{2}$ ».

Si la problématique du corps est devenue depuis les années soixante-dix un fort enjeu de la recherche transdisciplinaire en Occident, elle s'est développée plus tard en Asie orientale, ces toutes vingt dernières années, dans le sens de l'interrogation sur l'existence d'un « corps asiatique » spécifique, et ce selon quatre directions : les études foucaldiennes, féministes et culturelles ; l'anthropologie médicale ; les recherches sur la pensée néo-confucéenne et identitaire ; les développements du concept de body culture/culture du corps ${ }^{3}$. Du côté de la science de la littérature, les travaux sur la représentation du corps ont montré combien l'objet est aussi présent que mouvant, voire fuyant et difficile à cerner car il n'est souvent, sous son enveloppe apparente, que

1. Fragonard $2010: 352$. Les références des ouvrages en chinois et en japonais figurent en fin de chaque article composant le numéro.

2. Ce programme doit se clore en 2016-2017 par un colloque international avec publication des Actes.

3. Brownell $2009: 29-34$. 
« signe renvoyant à autre chose que lui-même ${ }^{4}$ », et « toujours disséminé dans un tissu de relations qui le dépassent ou le sous-tendent ${ }^{5}$ », relié à l'affirmation de quelque identité, ethnique, sociale, culturelle, intime.

Comme la problématique générale du corps en Asie orientale sous-tend le travail présenté ici sur le corps souffrant à travers les littératures locales, la question se formule en termes de mouvement et d'expression des corps dans un espace-temps pertinent, à savoir le Japon et la Chine continentale, que le choc de l'intrusion de l'Occident amène, voire oblige, à se réformer. Il s'agit, en l'occurrence, d'une modernisation entre tradition et modèles occidentaux, adhésion volontaire et résistance nationale, groupe et sujet émergent, qui cherche à adapter et adopter les savoirs, les pratiques, les idéologies et les technologies d'importation qui touchent à ce corps. Autant qu'une autre forme de discours, l'art de la littérature, lui-même soumis à mutation - ne seraitce que de langue -, rend compte du devenir du corps et des corps à maints niveaux, de l'anatomique au métaphysique en passant par le national.

À l'orée du XX' ${ }^{\mathrm{e}}$ siècle, avec l'expansion ultra-marine de l'Occident, l'Asie orientale subit le choc des forces et des connaissances qui bouleversent ses repères et l'obligent à opter pour une mutation conçue comme salutaire. Le corps est au carrefour des stimuli qui le balisent, le verbalisent, l'enlisent aussi parfois. De ce corps qui devient enjeu à l'échelle de la nation et bientôt de l'individu, de ce corps qui s'impose peu à peu face au sujet, la littérature, elle aussi soumise à mutations - notamment avec la modernisation de la langue : baihua chinois, genbun itchi japonais -, s'attache à rendre compte, autant que d'autres discours, au fil d'une histoire à rebondissements et reconfigurations multiples.

Pour aborder le corps souffrant, l'approche littéraire recourt à une philologie renouvelée, qui lui fournit la première base d'acception du sens syntagmatique, sans rien anticiper de son irisation textuelle et de la multiplicité des interprétations possibles. Elle recourt aussi aux sciences humaines, la philosophie et la sociologie au premier chef, l'histoire et la géographie culturelle ensuite, en tant qu'elles peuvent lui apporter le contexte et un substrat conceptuel, qui viennent renforcer l'analyse littéraire proprement dite, fondée sur la microlecture socio-poétique des textes.

Bien que l'expression linguistique du corps varie selon l'aire culturelle et au sein d'une même aire, bien que des concurrences puissent apparaître entre corps chinois et corps japonais, le corps sino-japonais peut corroborer sans

4. Touret $2014: 1$.

5. Prost $2010: 8$. 
mal, mutatis mutandis, le postulat global d'un « corps asiatique », réductible à nul autre, et notamment opposable au corps occidental. Cela dit, non sans paradoxe, la mondialisation est inhérente à ce « corps asiatique » qui, amené à se réinventer, doit parfois composer jusque dans sa chair avec l'Autre occidental. Il importe de préciser que si, comme nous le rappellent les études postcoloniales, l'expression de ce « corps asiatique »s'est bien déployée dans l'imagination exotique occidentale, ici notre lecture se construit à partir des textes eux-mêmes et des auteurs qui nous racontent une certaine histoire du corps, écrite depuis l'autre rive. C'est bien leurs propositions que nous analysons, sans déterminisme culturel ni rémanence orientaliste.

Plus précisément, pour dire le corps, le chinois ancien offre des termes voisins qui recoupent ce que les langues européennes - pour en rester à ce parallèle - entendent par corps : "xing signifie plutôt la forme actualisée, shen plutôt l'entité personnelle, le moi individuel, et $t i$ plutôt l'être constitutif. Aucun de ces termes ne coïncide tout à fait avec la notion européenne parce qu'ils répondent eux-mêmes à des termes divers et parce que, fonctionnant en binôme, ils s'éclairent également à partir de leur vis-à-vis ${ }^{6}{ }^{\prime}$. L'ensemble s'entend en rapport (à la fois d'opposition et de complémentarité) avec la dimension transcendante-animante (shen) précédant toute actualisation ; "l'entité personnelle » va de pair avec la fonction de conscience morale et la connaissance du cœur-esprit (xin), qui la régit ; " "l'être constitutif" a pour partenaire le souffle-énergie ( $q i)$ dont il est la matérialisation par condensationconcrétion $^{7}$ ». À l'époque moderne, le terme évolue en fonction des enjeux sociaux de la période considérée, notamment le binôme " corps-personne » (shen) et « cœur-esprit » $(x i n)^{8}$. Les termes xing, ti, et shen tendent à désigner le «corps » comme expression extériorisée de la personne et selon qu'il est plus ou moins perçu sous l'angle de l'objectivation ou du corps propre ${ }^{9}$. Le champ lexical du corps humain est très riche sur les rapports avec la personne (shen), avec sa matérialité et sa corporalité (tipo, tige, tili), ou sa carnalité, voire sa sensualité (routi), ainsi que pour la vie intérieure et les émotions du sujet. Ce lexique s'accroît dès la fin du XIX ${ }^{\mathrm{e}}$ siècle de l'apport de xénismes, de termes étrangers, ainsi que de termes anciens resémantisés à la faveur de l'apparition de nouveaux phénomènes. Au tournant du $\mathrm{XX}^{\mathrm{e}}$ siècle se développe aussi l'idée du corps comme une « carcasse » (qu,quke), inerte et dépersonnalisée, qui fait obstacle à l'émancipation des citoyens d'une nouvelle nation à construire,

\footnotetext{
6. Jullien $2005: 68-69$.

7. Jullien $2005: 69$.

8. Brownell 2009 : 34-37; Elvin 1989.

9. Link $2001: 46-51$.
} 
comme chez le réformiste Liang Qichao (1873-1929), et qu'il convient donc de dresser ; cette représentation dualiste ne restera pas étrangère à la littérature des années 1920.

Quant à la souffrance, le terme japonais de kumon, souci, ennui ou morosité à l'origine, passe ou plutôt revient en chinois avec le sens nouveau de souffrance romantique existentielle, en même temps que le lexique clinique des maladies nerveuses, telle la «neurasthénie » (shenjingshuairuo). Parmi les troubles intérieurs, un mot comme fannao est plus que fréquent dans la littérature chinoise des années 1920 , avec le sens de « tourments » et une nuance werthérienne. Les composés formés de teng ou tong peuvent déployer, eux, des connotations autant physiques que morales, bien que le sens premier soit corporel. Certains mots sont composés à partir de $j i$, « maladie », qui exprime par dérivation la souffrance ou le mal - tel jiku, « souffrances, malheurs », avec « $k u$ » pour ce qui est « pénible » car « amer » (voir la transcription moderne de kuli pour « coolie »). Le lexique de la blessure ou cicatrice (hen, shanghen), de la faiblesse (ruo, shuai), de la maladie (bing, ji) complète cet inventaire qui, en littérature chinoise moderne, exhibe une souffrance avant tout spirituelle, psychologique et mentale.

La langue japonaise, qui hérite du chinois - tout en ayant conservé son propre fond lexical -, dispose d'au moins quatre mots : shintai (sino-japonais), le corps au sens froidement objectif ; shin (sino-japonais)/mi (japonais), ou soimême, avec une distance réflexive et une connotation corporelle et physique, mais non psychosomatique ; karada (japonais), ou corps organique ; sei (sinojaponais), qui peut traduire l'idée de nature en tant qu'état inné et originaire des choses, ainsi que le sexe ${ }^{10}$. Le préfixe kara de karada se réfère à l'enveloppe, à la coquille, voire au vide, par opposition à mi, qui dénote le contenu - qu'on pense au jaune ou au blanc d'œuf : kimi et shiromi. Karada, utilisé jusqu'au XVII ${ }^{\mathrm{e}}$ siècle au moins pour désigner le cadavre, produit un effet visuel à la lecture selon qu'il est écrit en kanji ou en hiragana, donnant une impression beaucoup plus sensible dans le syllabaire. À ces quatre vocables peuvent s'ajouter d'autres termes, en sino-japonais ou en japonais, tels nikutai, pour l'expression du corps purement charnel, et les réfléchis, jibun, pour dire « soi(même) », et jishin, qui inclut le caractère shin (corps). Pour la souffrance, les termes de base sont surtout nayami et kurushimi ; kunô et kumon sont aussi fréquents pour signifier plutôt la souffrance intellectualisée, avec une gradation de nayami à kunô puis à kumon; kutsû existe également, mais se rapporte davantage à la douleur.

10. Legendre $2013: 298-299$. 
Il n'est ainsi que de constater l'étendue lexicale des termes liés au corps et à la souffrance, un champ de surcroît amplifié par la transdisciplinarité associée à l'approche littéraire. L'examen philologique informe le sens du mot, mais ne préjuge pas de sa production de sens en langage, objet de la praxématique ${ }^{11}$, seule à même de régler le mot en contexte, de lui donner sa portée en tant que construction sociale et vision du monde. Et c'est à la littérature et à la linguistique qu'il revient de déterminer le réglage du praxème.

La philosophie est d'un apport précieux en ce qu'elle aide à dépasser, dans l'approche du corps, la polarité classique, voire stéréotypée, qui oppose au corps-esprit de l'Asie orientale la division cartésienne du corps et de l'esprit en Occident. Opposition toute relative, d' ailleurs, qui tend à s'estomper avec le processus d'alignement sur ses propres normes et modèles que ledit Occident, dans la seconde moitié du XIX ${ }^{\mathrm{e}}$ siècle, impose plus ou moins à la Chine et au Japon, mais aussi avec l'orientalisation de l'Occident plus présente qu'en apparence ${ }^{12}$. Une opposition toutefois assez forte ici ou là pour informer les représentations immanentes aux textes.

Et c'est à la phénoménologie qu'il revient, surmontant le clivage corpsesprit, d'opposer la théorie du corps subjectif à celle de la subjectivité pure et désincarnée de Kant et de lier le corps-effet au moi-cause sans remonter discursivement de l'effet extérieur à la cause intérieure. Le philosophe Michel Henry définit le corps subjectif comme un mouvement subjectif qui n'est pas intermédiaire entre l'ego et le monde car il est intentionnalité ou volonté intentionnelle : «Ego, corps, mouvement, [...] ne font qu'une et même chose $^{13}$. » Il n'y a pas d'en-soi du corps. En ce sens, la parole, qui remanie les émotions, peut être une bénédiction autant qu'une malédiction, et la violence verbale, devenir un mode de l'intersubjectivité des corps. Ici, le mouvement subjectif unifiant, qui deviendra la chair dans la philosophie tardive de Michel Henry, s'accorde avec la nature historiquement mouvante d'un objet comme le corps, qui se décline aussi dans et par la progression du récit.

La philosophie apporte encore d'autres arguments non négligeables pour définir le corps souffrant. Paul Ricœur distingue ainsi douleur physique et souffrance psychique, en déclinant le souffrir selon deux axes : l'axe soiautrui, où le corps, par degrés, se perçoit à vif, endure la douleur physique, peine à la communiquer, la subit de la part d'autrui et finit par se sentir élu par elle ; l'axe agir-pâtir, où le corps se trouve dans l'incapacité de dire, de se

11. Lafont 1978.

12. Goody 1999.

13. Henry $1965: 83$. 
dire, d'agir, est donc diminué, mais c'est en parvenant à cet état de crispation que le sujet se met en état de décharger la parole qui peut le libérer. De même que le sujet se dit et s'affirme identitairement par l'expression de sa souffrance - en psychanalyse -, de même en littérature, mais à une nuance près, l'écriture est celle du non-dit, voire du silence sur la douleur éprouvée : d'où le recours à la litote, l'allégorie ou la métaphore. Les modes ou niveaux de construction textuelle du corps vont de la sensation sensori-motrice intersubjective à l'incorporation textuelle au moyen de quelque symbole. En fin de course, dans la régie littéraire du corps souffrant entre dire, vouloir dire, ne pas pouvoir dire et ne pas vouloir dire, c'est bien à la littérature qu'il revient de négocier cette tentative de verbalisation et, par là, de dire autre chose, entre quête de vérité et accomplissement esthétique - plutôt que ce qu'en disent les autres voies et disciplines du savoir et de la création.

De ces éléments définitionnels, d'ordre historique et philosophique, la critique littéraire peut tenir compte ou pas. Certes, elle ne peut pas ne pas croiser les sciences humaines et la philosophie, elle peut même adopter certains de leurs points de vue, mais elle peut aussi procéder par induction, interroger le tissu textuel avec ses propres outils. Par exemple, le style kinésique, repérable dans le texte à la dénotation d'un geste ou d'une mimique du personnage, expression parfois difficile à imaginer pour le lecteur mais significative, peut être rapporté à l'un des enjeux, voire à l'enjeu principal d'une œuvre ${ }^{14}$. Le caractère intentionnel du mouvement implique, à partir du réflexe sensorimoteur, palpable par autrui, une dynamique intersubjective, inscrite dans la trame textuelle, repérable dans l'enchaînement des actions.

Ce qu'apportent les sciences humaines ne saurait infléchir, sauf à enfermer le texte littéraire dans une grille, la capacité de l'imagination poétique à renouveler l'approche du corps souffrant et à ouvrir - ne serait-ce qu'à partir d'un hapax apparent - des pistes inexploitées et donc à sonder. C'est sans doute l'originalité de la littérature que d'embrasser le réel autrement que par pur reportage, de le dépasser pour le subvertir, pour l'anticiper, pour ajouter du monde au monde. Par là, elle touche à des zones inouïes, pertinentes ou moins pertinentes, mais qui ouvrent d'autres possibilités d'approche de ce réel. Mais si l'analyse littéraire est susceptible, travaillant sur un matériau autre que le document historique ou sociologique, de déboucher sur des résultats inédits, il convient de les comparer, à terme, avec ceux des autres disciplines. Ainsi parviendra-t-on à une approche plus substantielle de l'histoire du corps souffrant dans tous ses états...

14. Bolens 2008. 
L'originalité du projet tient à son approche en étendue : la Chine, le Japon, et en corpus : canonique et populaire. Voilà deux aires, deux littératures nationales qui, après avoir vécu sur une image sensiblement identique du corps, voire du corps malade, affrontent, non sans un décalage socio-économique, le cortège des nouvelles empiricités, et expérimentent d'autres modes du corps, entre recompositions des anatomies (mannequins, etc.) et constructions de corps robotisés (cyborgs, androïdes, clones) ou virtuels, qui se confrontent aussi avec l'émergence de la notion de sujet.

L'enjeu de cette recherche concerne autant la spécificité de la représentation du corps souffrant en Asie Orientale que celle de chacune des littératures visées, de Chine et du Japon. Dans quelle mesure leurs représentations littéraires de la corporéité en souffrance - entre le dedans et le dehors, le physique et le mental, la nature et la culture, le réel et le virtuel - se rejoignent-elles, et pourquoi ? Comment articulent-elles l'incorporation lettrée du corps en mal de luimême avec le corps du texte inscrit dans un corps social en proie à d'autres malaises, certes, mais aussi en quête d'équilibre ou d'une certaine esthétique ? Le processus d'hybridation et de métabolisation socio-culturelle, suscité par la réponse à l'étranger, est-il de la même nature sur la scène littéraire chinoise et japonaise, mais aussi sur d'autres ailleurs littéraires à l'heure de la mondialisation ? L'hypothèse de travail est qu'on passe d'une extériorisation du corps, produit d'importation, à son intériorisation subjective, mais que la pression des stimuli extérieurs - de la biopolitique en particulier - induit une tendance oscillant entre normalisation et esthétisation des corps en régime de souffrance...

Le même mouvement de spectacularisation du corps souffrant se retrouve partout. Entre pure et simple dénotation du corps et sa constitution en formesens (métaphore, allégorie, prosopopée, etc.), on passe, degré par degré, d'un corps absent ou présent en creux à un corps présent et pressant, qui compromet le sentiment qu'a le sujet (ou le groupe, pour le corps national) de son intégrité, de ce qui le constitue en tant que tel, et l'amène à réagir à la douleur et à la souffrance, c'est-à-dire à leur faire obstacle ou à les exploiter, toujours avec un ou des sens à la clé. Le rapport entre le genre littéraire et le mode d'écriture du corps importe à l'affaire. Alors que le corps est le plus souvent implicite au récit et n'a pas à être dit - il va de soi que la personne, l'actant si l'on veut, dispose d'un corps, faute de quoi pas de récit et pas d'identification possible du lecteur au personnage -, il est des genres axés sur la spectacularisation du corps, sur l'effet dramaturgique en somme - avec primat de l'hypotypose -, et ce à plus forte raison quand ce corps est en souffrance. C'est le cas du roman policier et du roman de cape et d'épée, ainsi que du théâtre, où le montreur, le corps exhibé et le public voyeur font bon ménage. Mais ce spectacle ne cesse d'osciller entre vérité et esthétique. 
Du point de vue de l'évolution historique, le corps réagit d'abord comme il peut au choc de l'intrusion de la machine occidentale. Le malaise du corps se fait alors symptôme d'un mal de civilisation. Lu Xun pâtit du spectacle du corps démembré de ses compatriotes. Nagai Kafū (1879-1959) affecte d'une fièvre typhoïde le jeune héros de La Sumida (1911), dont le corps ne peut pas plus se plier aux exercices de gymnastique imposés par l'État de Meiji qu'à la mort annoncée de ce lieu des traditions qu'est la ville basse - shitamachi - à Tôkyô.

Mais la littérature se renouvelle aussi par l'exploitation proprement dramaturgique des nouvelles facettes du corps introduites en partie par la science occidentale, la scientia sexualis, sans renoncer pour autant à l'ars erotica. Ici, le récit de cape et d'épée de la Chine exhibe à souhait la souffrance de corps soumis au supplice, de corps dont la douleur physique est à hauteur de la noirceur morale du personnage qui la subit. Là, le polar japonais joue à découper les corps et à les recomposer à des fins d'esthétique, et, de la sorte, à susciter par empathie un mélange de souffrance et de jouissance auprès du public, et ce non sans quelque lien avec le cadavre exquis des surréalistes ou certaines techniques locales de composition poétique à plusieurs.

Ilest vrai que, dans le cas japonais, la pathologie et même la psychopathologie de l'Occident sont passées par là et que le sadomasochisme trouve un terreau, qui lui permet de prendre du champ. Tout l'œuvre de Tanizaki Jun.ichirô (1886-1965) en prend acte. De même l'émergence du sujet à l'occidentale, avec l'amour dit romantique à la clé, est-elle à prendre en compte, car le naturalisme japonais, choisissant l'introversion plutôt que le discours social, exploite la dissection de ses tourments et place la chose sexuelle au centre de ses préoccupations.

La même insistance sur la maladie romantique, la subjectivité maladive et rongée de névroses dues à la frustration, notamment sexuelle, est développée par des auteurs chinois à partir du début des années 1920, en particulier Yu Dafu (1896-1945) ou Guo Moruo (1892-1978), qui ont découvert au Japon « roman du moi » et fantaisies macabres, ainsi que la théorie de Kuriyagawa Hakuson (1880-1923) sur la création comme expression symbolique de la frustration et de la mélancolie, thème qui sera au centre de la vie littéraire chinoise jusqu'à la moitié des années 1920. Quant à la thématique «décadente » d'un Tanizaki, elle continue occasionnellement à nourrir l'imaginaire littéraire chinois jusque dans les années 1940. Ces thématiques d'importation sont assimilées par les auteurs chinois, qui trouvent ainsi matière à exprimer leur propre discours de contestation de la famille, de la tradition, mais aussi de recherche d'affirmation nationale, et le thème libidinal se double alors ici de celui de la frustration en particulier face au Japon et à sa modernité. 
À ce stade, il semblerait que la littérature de Chine ait pour enjeu le corps national plutôt que le corps individuel en souffrance ou, du moins, qu'elle ait eu souvent des difficultés à les dissocier l'un de l'autre et, de fait, elle évolue dans le sens d'un réalisme socialiste au service du peuple avant de céder le pas à une littérature des cicatrices, qui dresse le bilan effrayant de la Révolution culturelle. Mais la littérature du Japon, sensible aussi aux idéologies d'importation, produit une prose prolétaire sui generis, où le corps ploie jusqu'à la mort sous l'exploitation capitaliste, comme dans Le Bateauusine (1929) de Kobayashi Takiji (1903-1933).

De la période de la République (1911-1949) jusqu'à nos jours, la Nouvelle littérature chinoise est en quête de l'expression qui rend sa voix à l'individu, au peuple, à la nation, aux victimes des violences politiques et historiques du $\mathrm{XX}^{\mathrm{e}}$ siècle : ainsi, un Mo Yan (1955-) cherche à démonter ou subvertir les récits officiels par la parole locale, un Han Shaogong (1953-) à réinventer une parole primitiviste. La littérature, républicaine en particulier, s'inspire autant de la pièce du dramaturge soviétique Sergueï Mikhaïlovitch Tretiakov (1889-1937), Hurle Chine! (1926) ${ }^{15}$, que de la gravure expressionniste de Munch ou du jeu «biomécanique » de Meyerhold pour produire le cri de l'éveil ${ }^{16}$. D'ailleurs, dès les années 1900, via les étudiants chinois au Japon, le théâtre moderne rejette la gestuelle et le chant codifiés et stéréotypés de l'opéra traditionnel au profit du " théâtre parlé » sur le modèle occidental : c'est la parole et la langue nationale nouvelle contre le corps hiératique du passé. L'élite aux idées modernes rejettera ensemble l'opéra classique «barbare »; le grand acteur d'opéra Mei Lanfang (1894-1961) sera accusé par un révolutionnaire comme Chen Duxiu (1879-1942, l'un des fondateurs du PCC) de se complaire à jouer des rôles féminins devant les spectateurs japonais. Cette affirmation obsessionnelle du rôle masculin pour redonner une voix virile à la Chine affaiblie s'exprime par exemple dans le roman populaire best-seller de 1941, Bégonia (Qiuhaitang) de Qin Shou'ou (1908-1993), plusieurs fois adapté au cinéma, en théâtre, en série TV jusque dans les années 2000 : un jeune acteur de l'opéra chinois, au visage défiguré par un seigneur de la guerre, veut passer de l'interprétation de rôles féminins à un rôle et à un chant masculins ${ }^{17}$. En ce sens, la parole de la littérature chinoise moderne est en grande partie une plainte et un cri rentré de souffrance.

Plus près de nous, même si la littérature chinoise se dégage peu à peu du carcan idéologique et parvient à inventer un sujet individuel à sa façon, elle

15. Рычи, Китай [rychi kitai].

16. V. Tang $2008: 213-227$.

17. V. Wang 2003 et Braester 2000. 
n'a pas fini pour autant de régler ses comptes avec la Révolution culturelle. L'écrivaine Chi Li (1957-), qui fait la transition entre la Chine de Mao et celle d'après, orchestre une confrontation conjugale cruelle dans Pour qui te prendstu ? (1995), entre le mari cadre du Parti et la femme qui découvre qu'il est à la source de tous les maux dont a été victime sa famille.

Au Japon aussi, la femme réagit aux violences infligées par le corps national, notamment durant la guerre d'Asie-Pacifique, sans doute avec une latitude de manœuvre plus ample qu'en Chine, dans le sens de la remise en question de la virilité nationale d'abord atteinte par la défaite - comme dans cette scène du Pavillon d'or (1956) de Mishima Yukio (1925-1970) où un GI bon teint force un jeune bonze complexé à donner des coups de pied dans le ventre d'une geisha... enceinte -, puis laissée pour compte, en pleine période de haute croissance économique. Pour sa part, Kôno Taeko (1926-2015) met en scène la souffrance du corps féminin au profit de son auto-affirmation, face à la mémoire de la guerre confisquée au profit des hommes.

La série d'articles proposée ici scrute le corps souffrant, malade et déformé, prégnant dans le corpus littéraire chinois et japonais de la fin du XIX siècle jusqu'au début des années 1990, dans ce monde où le sinogramme, longtemps dénoncé comme obstacle à la transformation, retrouve la vie propre qui était la sienne en Chine pré-moderne, et incarne désormais la volonté culturelle chinoise de remporter les défis de la modernité ${ }^{18}$. Les mutations consécutives aux contacts avec l'Occident entraînent volens nolens le réajustement généralisé du corps qui, tiraillé entre us anciens et nouveaux, pâtit dans sa santé, dans ses sens, dans son identité. La littérature d'Asie orientale ne se contente pas de mimer ce mal-être en tous ses états. Elle cherche aussi parfois à le dire avec une écriture qui fait littéralement corps avec son objet et laisse émerger une autre figure du sujet. L'ensemble offre un panorama qui combine la mise en mouvement des corps jusqu'à leur éventuelle mutation sous les formes les plus diverses, leur orchestration émotionnelle entre les instances de représentation (auteur/montreur, corps montré/montrant, public/lecteur ému), leur sémantisation socio-culturelle en fonction des modes et des besoins.

1. Emmanuel Lozerand s'attache, dans « Corps, maladie, écriture chez trois auteurs japonais du début du XX' $\mathrm{X}^{\mathrm{e}}$ siècle : Nakae Chômin, Masaoka Shiki, Natsume Sôseki », à trois œuvres singulières publiées entre 1901 et 1911, mal classables, élaborées à partir d'une situation de maladie. Nakae Chômin, atteint d'une tumeur cancéreuse à la gorge, se lance en toute hâte dans Un an et demi, la durée même du temps que les médecins lui accordent à vivre ; Masaoka Shiki,

18. Voir Bachner $2014: 1-14$. 
souffrant d'une tuberculose osseuse douloureuse et invalidante, donne jour après jour, au quotidien Nihon, Une goutte d'encre depuis son Lit de malade [de] six pieds de long ; Natsume Sôseki, rescapé d'une hémorragie gastrique, publie en feuilleton, dans le journal Asahi, Choses dont je me souviens pour garder la mémoire des sensations complexes qui furent les siennes dans les semaines écoulées. Tous trois refusent la métaphorisation de la douleur, qu'elle soit stigmatisation ou esthétisation, et inventent des écritures quasi humorales, pour ouvrir depuis leurs corps malades des fenêtres sur le monde.

2. Victor Vuilleumier met à jour, dans « Le corps souffrant chez Lu Xun : allégorie muette de l'obstacle et appropriation de la modernité », le complexe édifice de l'allégorisation du corps souffrant chez Lu Xun. Le corps n'est en effet que très peu décrit. Cette absence permet de dire indirectement une souffrance multiple : celle de la honte ressentie au spectacle de la crise historique traversée par la Chine, honte imputable à la nature nationale, coupable de porter une culture «cannibale » et complice de sa perpétuation, ou simplement celle de la mélancolie existentielle. Le corps incarne le signe de la tradition, qui fait obstacle à l'émancipation de l'individu. Libérer la voix vive de la nation chinoise impose et de détruire symboliquement le corps reçu en héritage et de subvertir l'esthétique et les paradigmes scientistes de la corporalité et de la puissance élaborés par la modernité chinoise mondialisée. Le vrai médecin agit par l'écrit sur l'esprit, la culture, les lettres, non sur le corps ou la civilisation matérielle. Valoriser le cœur sur le corps permet de fonder un projet de modernité, en métaphorisant la médecine occidentale et japonaise, reine des sciences modernes. L'étiologie du corps chinois souffrant confère une fonction thérapeutique à la littérature.

3. Gérald Peloux explore pour sa part, dans « Cadavre vivant et pantin désarticulé : souffrance et reconfiguration des corps dans l'œuvre d'Edogawa Ranpo », le savant dispositif de spectacularisation du corps souffrant dans les récits policiers d'Edogawa Ranpo. La surabondance de corps, surtout féminins, démembrés, reconfigurés, y reconduit certains canons esthétiques des années vingt et trente, basés sur le grotesque, l'érotisme et l'absurde. Malgré la violence qui traverse ces histoires, la description du corps souffrant y est étonnamment absente, trait qui signale le désir auctorial de détourner les techniques et stéréotypes du genre policier pour proposer, là même où l'on attendrait une souffrance exhibée, une approche originale, quasi interactive, du rapport entre auteur, texte et lecteur - comme le montre l'examen de « la Chenille » (1929), clé d'une œuvre ambivalente au regard de la souffrance physique.

4. Nicolas Zufferey analyse, dans « Corps fort et corps blessé chez Jin Yong et dans quelques romans d'arts martiaux chinois contemporains », certaines 
représentations fictionnelles de la souffrance du corps. Cette littérature privilégie le plus souvent le corps de l'homme fort, de surcroît apte à réguler ses énergies internes au moyen de techniques de travail sur le souffle. Mais dans Aventurier fier et souriant, œuvre du célèbre romancier Jin Yong, le corps souffrant n'est plus seulement celui du vilain, que le champion défait, mais aussi celui de ce dernier, quand des énergies contraires se combattent en lui et le font souffrir. Le corps souffrant du héros peut être interprété comme un corpsmonde, qui aspire à une vie pure et simple, dans une Chine pacifiée, mais que traversent les tensions énergétiques, lesquelles reflètent les luttes politiques du moment - Aventurier fier et souriant, écrit au début de la Révolution culturelle, est aussi une dénonciation des dérives politiques de l'époque maoïste.

5. Xu Shuang et Ariadna de Oliveira Gomes retracent, dans « Le corps souffrant dans la littérature chinoise depuis la Nouvelle période (1979-2015) », les expériences du corps souffrant au fil des programmes politiques impulsés par le régime communiste depuis Mao. Le sujet, mis en demeure mais aussi désireux de se plier aux exigences de la foi doctrinale, se fait docile aux dépens de son corps, comme l'illustre la littérature dite « des cicatrices », apparue à la fin des années soixante-dix. Il sort brisé, impuissant, affolé de cette épreuve, ou s'évade dans la mort. Mais le passage à une nouvelle économie politique le met aux prises avec une nouvelle géhenne. Et c'est seulement dans les années quatre-vingt-dix qu'émerge un corps chinois relativement plus libéré des contraintes idéologiques.

6. Gérard Siary s'attache, dans « Corps sensible et corps pratico-inerte : femme frustrée et kamikaze mutique dans une nouvelle de Kôno Taeko, "Tetsu no uo" ("Poisson de fer") », à ferrer le poisson de la nouvelle de Kôno Taeko, un poisson de fer qui symbolise ici la torpille à guidage humain (kaiten) pilotée par les tokkôtai.in ou kamikazes, nef sous-marine exposée au sanctuaire Yasukuni de Tôkyô, mausolée des soldats péris à la guerre et devenus des dieux. La protagoniste, dont le mari s'est fait exploser dans l'engin en question, s'y engouffre de façon clandestine afin de s'approprier cet homme qu'elle a peu connu et qui l'a maintenue dans le silence jusqu'à très tard dans son existence. La nouvelle dit le traumatisme de la guerre au féminin, autant la mort du sujet militaire que la souffrance du corps féminin, qui se voit ravir son existence affective au bénéfice de l'historiographie officielle axée sur l'héroïsme guerrier.

Ces études montrent ainsi qu'au corps souffrant imposé en Chine, entre colonisation, oppression politique et appropriation volontaire des représentations de la faiblesse, tend à s'opposer le corps souffrant instrumentalisé au Japon, entraîné dans une course sans limites vers une modernisation rêvée. Au corps national mis à mal en Chine tend à s'opposer le corps national 
subverti au Japon. Et, de tous côtés, la guerre des genres modifie lentement les rapports de force, vers l'affirmation d'un corps féminin, dont la souffrance sert parfois d'identité ambivalente mais puissante. Insistons : ce ne sont là que des tendances. Mais elles pourraient bien renvoyer, du point de vue anthropologique, à quelque étiologie de la représentation de la souffrance, qui reste à interpréter en termes culturels.

\section{BIBLIOGRAPHIE}

BACHNER, Andrea (2014). Beyond Sinology, Chinese Writing and the Scripts of Culture. New York, Columbia University Press.

BoLENS, Guillemette (2008). Le Style des gestes : corporéité et kinésie dans le récit littéraire. Lausanne, Éditions BHMS.

Braester, Yomi (2000). « Revolution and Revulsion : Ideology, Monstrosity, and Phantasmagoria in Ma-Xu Weibang's Film Song at Midnight ». Modern Chinese Literature and Culture, vol. 12, $\mathrm{n}^{\circ} 1: 81-114$.

BROWNELL, Susan (2009). «The Global Body Cannot Ignore Asia ». In TURNER, Brian S. et YANGWEN, Zheng (2009). The Body of Asia. Berghahn Books : 23-39.

ELVIN, Mark (1989). «Tales of Shen and Xin : Body-Person and Heart-Mind in China during the Last 150 Years ». In FEHER, Michel (dir.), Fragments for a History of the Human Body. New York, Zone, t. 2 : 267-349.

FRAGONARD, Marie-Madeleine (2010). Corps sanglants, souffrants et macabres. Paris, Presses Sorbonne Nouvelle.

Goody, Jack (1999). L'Orient en Occident [The East in the West, 1996]. Paris, Seuil.

HENRY, Michel (1965). Philosophie et phénoménologie du corps. Paris, PUF.

Jullien, François (2005). Nourrir sa vie à l'écart du bonheur. Paris, Seuil.

LAFONT, Robert (1978). Le Travail et la langue. Paris, Flammarion.

LEGENDRE, Pierre (2013). Tour du monde des concepts. Paris, Fayard.

LINK, Gudula (2001). Leib und Körper, zum Selbstverständnis im vormodernen China. Frankfurt am Main, Peter Lang.

Prost, François et WiLgauX, Jérôme (2006). Penser et représenter le corps dans l'Antiquité. Rennes, PUR.

RICOEUR, Paul (1994). «La souffrance n'est pas la douleur ». Autrement, « Mutations », $\mathrm{n}^{\circ} 142: 58-69$.

TAng,XiaObIng (2008). Origins of the Chinese Avant-Garde. The Modern Woodcut Movement. Berkeley/Los Angeles/Londres, University of California Press.

TOUBLET, Cécile (2014). «Le corps comme signe ». Acta fabula, vol. 15, n 5, «Corpora corporis », fabula.org/acta/document5916, page consultée le 10 mai 2015.

WANG, David Der-wei (déc. 2003). «Impersonating China ». Chinese Literature : Essays, Articles, Reviews, vol. $25: 133-163$. 
Cécile Sakai, Gérard Siary et Victor Vuilleumier

\section{GLOSSAIRE CHINOIS}

baihua 白話

bing 病

Chen Duxiu 陳獨秀

fannao 煩惱

Guo Moruo 郭沫若

Han Shaogong 韓少功

hen 痕

$j i k u$ 疾苦

kuli 苦力

Liang Qichao 梁啓超

$q i$ 氣

Qin Shou'ou 秦瘦鷗

Qiuhaitang 秋海棠

$q u$ 軀

quke 軀殼

Mei Lanfang 梅蘭芳

Mo Yan 莫言

routi 肉體

shanghen 傷痕

shen 身

shen 神

shenjing shuairuo 神經衰弱

shenti 身體

teng 疼

$t i$ 體

tige 體格

$t i l i$ 體力

tipo 體魄

tong 痛

Yu Dafu 郁達夫

xin 心

xing 形 
Les épreuves du corps en littérature. Les cas de la Chine et du Japon

\section{GLOSSAIRE JAPONAIS}

Edogawa Ranpo 江戸川乱歩

genbun.itchi 言文一致

jibun 自分

jishin 自身

kaiten 回天

Kani.kôsen 蟹工船

kara 殼·空

karada 体・からだ

kimi 黄身

Kobayashi Takiji 小林 多喜二

Kôno Taeko 河野多惠子

kumon 苦悶

kunô 苦絁

Kuriyagawa Hakuson 厨川白村

kurushimi 苦しみ

kuts $\hat{u}$ 苦痛

Masaoka Shiki 正岡子規

Mishima Yukio 三島由紀夫

Nagai Kafû 永井荷風

Nakae Chômin 中江兆民

Natsume Sôseki 夏目漱石

nayami 悩み

nikutai 肉体

sei 性

shin/mi 身

shintai 身体

shiromi 白身

shitamachi 下町

Tanizaki Jun.ichirô 谷崎潤一郎

Tetsu no иo 鉄の魚 
